\title{
A chinese case of prevotella intermedia and streptococcus constellatus intracranial mixed infection
}

\author{
Shanying Mo ${ }^{1} \cdot$ Liuhua $\mathrm{Wei}^{1} \cdot$ Hongmou Chen ${ }^{2} \cdot \mathrm{Rui} \mathrm{Li}^{3} \cdot$ Shuping $\mathrm{Li}^{4} \cdot$ Guolan $\mathrm{Luo}^{1}$
}

Received: 11 May 2017 / Accepted: 23 October 2017 / Published online: 2 November 2017

(C) The Author(s) 2017. This article is an open access publication

\begin{abstract}
Streptococcal Species is increasingly recognized as a potentially preventable emerging infection in human's brain with high prevalence around the world. Streptococcus constellatus is one of the most common pathogens. Meanwhile, anaerobic bacteria are the rare causes for intracranial infection. To date, intracranial mixed infection caused by Prevotella intermedia and Streptococcus constellatus has not been reported. We reported a Chinese case to raise the global awareness of severity of the intracranial mixed infection. Here, we illustrated the epidemiological risk factors, clinical manifestations and outcomes of the patient. For patients who suffer from exacerbated brain infection with fetid cerebrospinal fluid, early repeated imaging is urgently needed and empiric antibiotic therapy should consider anaerobic and aerobic bacteria in these situations.
\end{abstract}

Keywords Prevotella intermedia $\cdot$ Streptococcus constellatus $\cdot$ Intracranial mixed infection $\cdot$ MRI $\cdot C T \cdot C h i n a$

Shanying Mo and Liuhua Wei contributed equally to this work.

Guolan Luo

luog12017@126.com

1 Medical Science Laboratory, the Fourth Affiliated Hospital of Guangxi Medical University, Liuzhou, Guangxi, China

2 Department of Neurosurgery, the Fourth Affiliated Hospital of Guangxi Medical University, Liuzhou, Guangxi, China

3 MRI Department, the Fourth Affiliated Hospital of Guangxi Medical University, Liuzhou, Guangxi, China

4 Department of Radiology, the Fourth Affiliated Hospital of Guangxi Medical University, Liuzhou, Guangxi, China

\section{Background}

Streptococcal Species is an increasingly recognized intracranial infectious pathogen. Streptococcus constellatus has been described as the fourth common clinical cause (Chang et al. 2002).There were increasing reports of brain infection caused by anaerobic bacteria, such as Prevotella oris and Peptostreptococcus micros (Frat et al. 2004), Parvimonasmicra (Ko et al. 2015), Peptoniphilus asaccharolyticus (Okui et al. 2016) and Polymicrobial anaerobic(Llitjos et al. 2016), although anaerobic culture of cerebrospinal fluid (CSF) was not recommended(Baron et al. 2013). "Spontaneous" form and "mixed" bacterial meningitis rarely appeared in middle-aged person, except in the post-neurosurgical (post-NS) patients(Tsai et al. 2012). To date, there have not been any cases about cerebral infarction accompanied by intracranial mixed infection caused by Prevotella intermedia and Streptococcus constellatus in China.

\section{Case report}

The patient, a 48-year-old previously healthy man, was sent to the hospital presented with no obvious cause for sudden rightsided weakness, chills and fever one day ago. Cranial CT showed the left frontal lesions with unknown cause. However, his illness was aggravating. He became unconscious and irritable, and then was rushed to the Fourth Affiliated Hospital of Guangxi Medical University for further treatment. The patient's families denied both he and his family had a history of hypertension, diabetes, coronary heart disease and other chronic or infectious diseases like hepatitis and tuberculosis etc. The patient's parents, siblings, and children were all physically fit, but the patient had been smoking and drinking for many years and had a history of alcoholic liver. 
On examination, he was confused and disorientated with a temperature at $40{ }^{\circ} \mathrm{C}$. Two pairs of aerobic and anaerobic blood cultures were performed immediately. He had a high blood pressure of 163/98 $\mathrm{mmHg}$ and meningeal signs, with tingling eyes open, no words, no action obeyed, tingling left limb visible activity and neck resistance $(+)$. Brain magnetic resonance imaging (MRI) revealed swelling of the left hemisphere, shallowing of sulcus and schizencephaly, abnormal lesions at the left frontal lobe and the left falx, narrowing of the left lateral ventricle due to compression and the midline structure shifted to the right (Fig. 1a-f). The cranial magnetic resonance venography (MRV) images showed abnormality in straight sinuses and superior sagittal sinus (Fig. 1g). The cranial magnetic resonance angiography (MRA) images suggested no obvious abnormalities (Fig. 1h). Results of blood tests and blood gas tests were shown in Tables 1 and 2. Results of hepatitis B virus (HBV) tests in time-resolved fluoro immune assay (TRFIA) were shown in Table 3. According to the
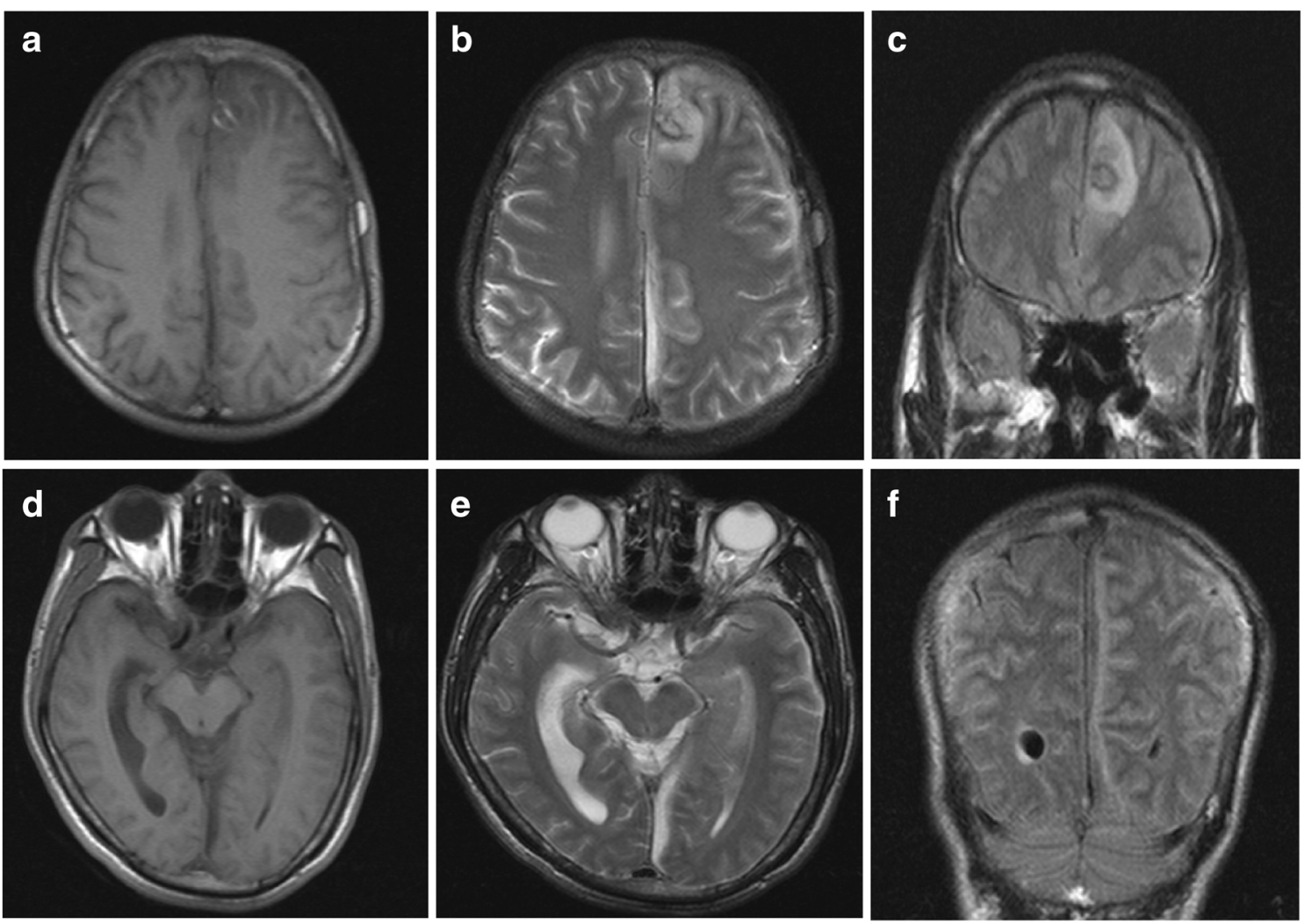

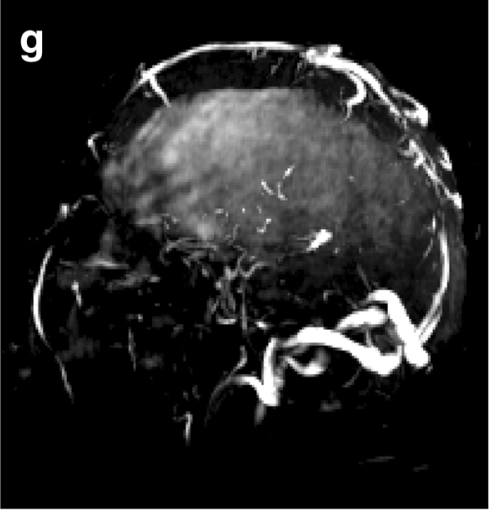

Fig. 1 a-f Brain MRI revealed the left hemisphere swelled, whose cerebral sulcus and schizencephaly shallowed, especially in cerebral cortex, with patchy signal intensity which was gyriform on brain T2weighted imaging (T2WI) and slightly high by fluid attenuation inversion recovery (FLAIR); the left frontal lobe, whose internal annular signal intensity was high on T1WI, low on T2WI and low on FLAIR was observed with patchy signal intensity that was slightly low on T1-

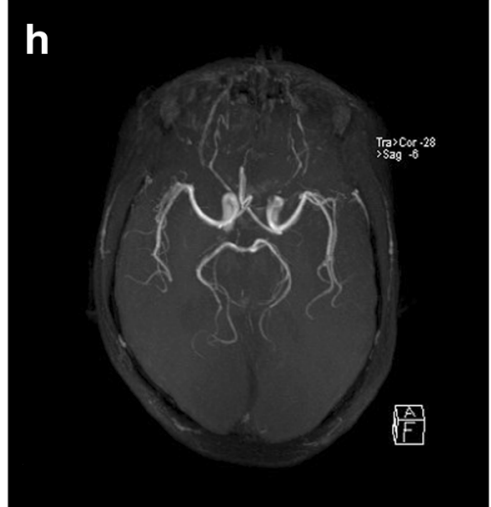

weighted images (T1WI), slightly high on T2WI and high on FLAIR; the left falx was observed with striped signal intensity that was low on T1WI, high on T2WI and low on FLAIR. $g$ MRV showed that straight sinuses were not clearly seen and superior sagittal sinus was not clear enough to be seen with rough cerebrovascular walls and focal luminal stenosis. $\mathbf{h}$ The cranial MRA showed no obvious abnormalities 
Table 1 Results of blood tests

\begin{tabular}{llll}
\hline Item & Day 2 & Day 3 & Reference interval \\
\hline White blood cell & 9.51 & 28.28 & $(3.97-9.15) \times 109 / \mathrm{L}$ \\
Neutrophils & 9.01 & 25.57 & $(2-7.5) \times 109 / \mathrm{L}$ \\
CRP & $>200$ & $>200$ & $<10 \mathrm{mg} / \mathrm{L}$ \\
\hline
\end{tabular}

above mentioned examinations, the patient was diagnosed with intracranial infection and started to take ceftriaxone sodium empirically.

On the second day, the patient complained of dizziness and headache without nausea. Due to unexplained cerebral infarction, enteric-coated aspirin was taken for anticoagulation and atorvastatin calcium for plaque stabilization. Lumbar puncture was performed to draw CSF. The CSF was with putrid odor and noted to be cloudy and soup-like with a protein level of $2433 \mathrm{mg} / \mathrm{L}$, a glucose level of $0.05 \mathrm{mmol} / \mathrm{L}$ (concomitant serum glucose was $7.97 \mathrm{mmol} / \mathrm{L}$ ), and $24.451 \times 10^{9}$ leukocytes /L (80\% neutrophils and $20 \%$ lymphocytes). CSF gram staining revealed gram positive cocci and gram negative bacilli. Cultured specimens were planted on the blood agar and chocolate agar for incubation at $37^{\circ} \mathrm{C}$ under aerobic and anaerobic conditions regarding the smell of CSF.

At 18:27 on the third day, blood gas test showed that type I respiratory failure, respiratory alkalosis and metabolic acidosis occurred in the patient with shortness of breath even if he was assisted by ventilator. At 22:55, the patient showed severe consciousness disorder and coma, with GCS3 points, disappeared light reflex. Brain herniation was considered, and the CT scan of head was taken immediately (Fig. 2). It showed that the patient had cerebral hernia and should receive craniotomy and decompressive craniectomy. At 23:48, bilateral mydriasis in the preparation of anesthesia appeared in the patient with his blood pressure of 30-40/10-20 $\mathrm{mmHg}$ and heart rate of 140 beats per minute, which had no surgical indications. Then the patient was sent back to the ward.

At 3:00 on the fourth day, the patient's family gave up treatment and asked to be discharged, even though the patient was still in the critical condition. After that, the colonies in CSF were identified as Prevotella intermedia and Streptococcus constellatus using matrix-assisted laser desorption ionization time-of-flight mass spectrometry (MALDI-

Table 2 Results of blood gas tests

\begin{tabular}{lllll}
\hline Item & Day 2 & Day 3-1 & Day 3-2 & Reference interval \\
\hline PH & 7.515 & 7.452 & 7.629 & $7.35-7.45$ \\
PCO2 & 24.2 & 30.3 & 13.8 & $35-45 \mathrm{mmHg}$ \\
PO2 & 113.7 & 119.1 & 58.7 & $80-100 \mathrm{mmHg}$ \\
\hline
\end{tabular}

Table 3 Results of HBV (TRFIA)

\begin{tabular}{lcl}
\hline Item & Quantitative results & Reference interval \\
\hline $\mathrm{HBsAg}$ & 0.000 & $0-0.2 \mathrm{ng} / \mathrm{ml}$ \\
$\mathrm{HBsAb}$ & $>640.000$ & $0-10 \mathrm{mIU} / \mathrm{ml}$ \\
$\mathrm{HBeAg}$ & 0.000 & $0-0.5 \mathrm{PEIU} / \mathrm{ml}$ \\
$\mathrm{HBeAb}$ & 0.560 & $0-0.2 \mathrm{PEIU} / \mathrm{ml}$ \\
$\mathrm{HBcAb}$ & 5.661 & $0-0.9 \mathrm{PEIU} / \mathrm{ml}$ \\
\hline
\end{tabular}

TOF MS) VITEK® MSMALDI-TOF MS (bioMériux) with a $99.9 \%$ probability. It took only four days from the symptom onset to treatment abandoning. Finally, the results of blood bacterial culture were negative.

\section{Discussion}

Adult bacterial meningitis ( $\mathrm{ABM})$ is a serious infectious disease of the central nervous system (CNS)(Chang et al. 2008; Durand et al. 1993). Unlike brain abscess, it is typically a mono-microbial infection (Ruef 2000). According to the criteria in the literature(Tsai et al. 2012), the case reported was classified as the "spontaneous" form and "mixed" bacterial meningitis (Chang et al. 2000). Mixed infection was not uncommon in post-NS ABM (Lai et al. 2013), but rarely appeared in "spontaneous" form caused by anaerobic and aerobic bacteria.

Streptococcus constellatus is generally a commensal organism found in the mouth, oropharynx, and gastro intestinal tract. This organism is not only cultured from dental caries and periodontal disease but also isolated from gastrointestinal perforations, obstetric infections, brain abscesses and meningitis (Chang et al. 2002; Piscitelli et al. 1992; Roca et al. 1998; Moller et al. 1999) .

Prevotella, previously classified in the genus Bacteroides, is a genus of an obligate anaerobic gram-negative rod-shape bacterium (Shah and Collins 1990). Many species of the genus Prevotella are pathogens that cause oral diseases. Prevotella intermedia is known to cause various oral disorders such as periodontal disease (Mombelli et al. 2000) and periapical periodontitis(Jacinto et al. 2003), colonize in the respiratory tract and is associated with cystic fibrosis and chronic bronchitis (Brook and Frazier 2003) and abscesses in the head and neck spaces (Bancescu et al. 2015), as well as meningitis(Brook 2003).

Prevotella strains and Streptococcus constellatus were reported to be isolated from pus specimens in abscesses in head and neck spaces in a Romanian study (Bancescu et al. 2015). Pin-Chieh $\mathrm{Wu}$ et al. reported in 2014 that Prevotella brain abscesses accompanied by stroke would occur after dental extraction in a young patient (Wu et al. 2014). For the 48year-old patient in this case, no dental extraction, dental 

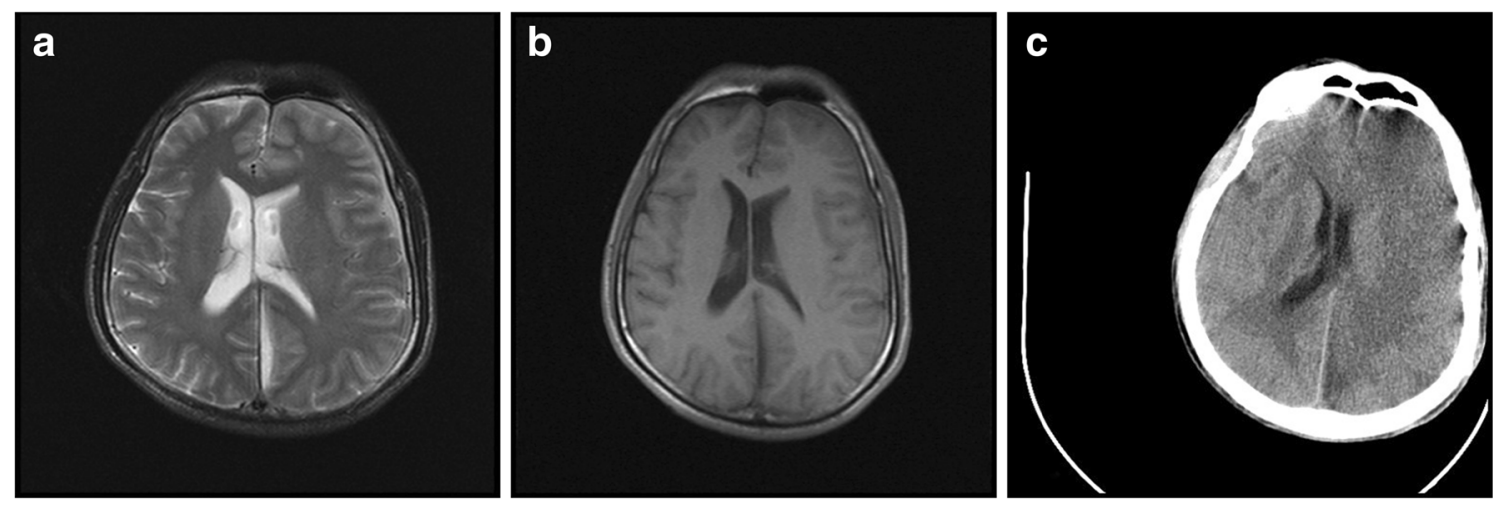

Fig. 2 Compared with the pre-treatment MR(Fig. 2a T2WI, Fig. 2b T1WI), the post-treatment CT (Fig. 2c) revealed aggravated swelling of the left cerebral hemisphere, especially in cerebral cortex, shallowing of

cerebral sulcus and schizencephaly, narrowing of the left lateral ventricle due to compression. The midline structure had shifted to the right obviously,which indicated cerebral hernia was developed carries or other oral diseases were reported in the case records. But their possibility as the cause to infection could not be ruled out since most adults had evidence of dental cavities (Contreras et al. 2010). On the other hand, no abscesses in head were reported in this case as well.

In a report from Pin-Chieh Wu (Wu et al. 2014), a 32-yearold healthy man with Prevotella brain abscesses and stroke following dental extraction experienced nine days of progressive headache and symptoms appeared 11 days later. Unlike the young man, the patient in this case suffered from rightsided weakness, chill and fever without any obvious causes and was immediately examined with sudden acute cerebral infarction and mixed infection caused by Prevotella intermedia and Streptococcus constellatus. The disease progression only lasted four days until his family abandoned treatment due to the exacerbation of the disease.

In a hospital-based study in Taiwan (Chang et al. 2008), diabetes mellitus (DM) was the most common underlying disease in patients with spontaneous infections, followed by liver disease, especially liver cirrhosis and alcoholism. In this study, the patient with spontaneous mixed infectious ABM had a history of HBV infection and had addiction to smoking and alcohol with years of alcoholic liver. Mixed infection was relatively few. Over a period of 10.5 years in a hospital, only 21 cases (totaling 52 strains) of mixed infection were reported and $86 \%$ of the mixed infected groups were classified as postNS type of ABM (Tsai et al. 2012).

Consistent with previous report (Llitjos et al. 2016), the patient's white blood cell(WBC) and protein level in CSF increased, while CSF glucose level decreased significantly with poor prognosis. ABM patients with mixed infection had a lower WBC count, lower protein and lactate concentration when compared to those with mono-microbial infection in the previous reported CSF study (Tsai et al. 2012). However, the leukocytes in patient in this case was $24.451 \times 10^{9} / \mathrm{L}$, which was significantly higher than those in the previous study, while the glucose showed the opposite result compared with those in the previous study (Tsai et al. 2012).

It might be a feasible way to combine gram staining with the characteristics and odor of CSF so as to identify meningitis with mixed infection of anaerobic bacteria preliminarily. Bacteroides fragilis concealed in an infant with Escherichia coli meningitis, and the apparent concealment of $B$. fragilis among E. coli in the CSF was possible because of their morphologic similarity; both organisms appeared as gram-negative, rod-shaped bacteria(Ganeshalingham et al. 2014). Frank pus was obtained from the $E$. coli and $B$. fragilis mixed infectious meningitis (Ganeshalingham et al. 2014). Foul smelling pus also appeared in the brain abscess due to Streptococcus spp. and Prevotella spp. (Sakamoto et al. 2009). For the patient in this study, lumbar puncture was performed to draw the soup-like CSF with putrid odor and gram staining revealed gram positive cocci and gram negative bacilli.

It might not be sufficient to use cefotaxime alone for meningitis with mixed infection with anaerobic bacteria. Antibiotic resistance was increasing not only in aerobic pathogens but also in anaerobic pathogens(Aldridge 1995). In anaerobes, production of $\beta$-lactamases is the most common mechanism of $\beta$-lactam resistance and is most frequently encountered in the $B$. fragilis group and the genus Prevotella with significant interspecies differences (Dubreuil et al. 2003). In an infant with $E$. coli and $B$. fragilis mixed infectious meningitis (Ganeshalingham et al. 2014), B. fragilis was confirmed resistant to ceftriaxone. In an in-vitro susceptibility test (Weintraub et al. 2016), Prevotella isolates were susceptible to metronidazole but resistant to ceftriaxone. It is well-known that metronidazole had a very good activity against most strict anaerobes, including Prevotella (Bancescu et al. 2015). Antimicrobial susceptibility testing of Prevotella spp. would take several days to complete and the delay in reporting the susceptibility results might have negative clinical implications for the patients(Sherrard et al. 2014). Metronidazole may still continue to be recommended in pyogenic diseases of head and 
neck but always in association with a drug active against aerobic bacteria, since such infections are usually mixed infections (Bancescu et al. 2015).

In diagnosis of cerebral infarction accompanied by meningitis with mixed infection, it is important for patients to receive imaging examination. Imaging of the brain and spine should be performed in a timely manner and reviewed specifically(Ganeshalingham et al. 2014). Brain CT scan and contrast-enhanced magnetic resonance images contributed to discovering cervical spinal epidural abscess and meningitis (Frat et al. 2004). Cranial MRA, MRI and head CT on the patient in our case showed cerebral infarction and whole brain swelling to reveal the signs of brain infection.

The patient was initially sent to the hospital for brain infarction, and then was diagnosed with intracranial mixed infection. At last, his family abandoned treatment. There might be several reasons for the exacerbation of the disease in such a short time. First, cerebral infarction aggravated; second, ceftriaxone used alone and Prevotella spp. might be resistant to ceftriaxone; third, fimbriae of Prevotella intermedia could induce hemagglutination (Leung et al. 1996), and then antibiotic resistance might accelerate the deterioration process of cerebral infarction.

Vancomycin plus a third or fourth generation of cephalosporin were the initial empiric antibiotics used in the treatment of patients with suspected ABM and the antimicrobial regimen was adjusted subsequently after the culture results were available (Tsai et al. 2012). When anaerobic bacteria was suspected to induce mixed infectious meningitis, empiric antibiotic therapy should consider anaerobic bacteria timely.

There were no previously reported risk factors expect a history of alcoholic liver and HBV infection for the patient. In this case, bacterial brain infection might appear as a cause to cerebral infarction when there was no trauma. "Spontaneous" form mixed infectious meningitis was rare, but it might be possible to occur. Anaerobic bacteria isolation was difficult, which usually required specific methods, and could be delayed (Frat et al. 2004). It should be noted that anaerobic culture should be added for the stench or cloudy CSF.

\section{Conclusion}

This case demonstrates the possibility of cerebral infarction accompanied by mixed infectious meningitis. The clinical and laboratory indicators of ABM with mixed infection are not distinctive. In summary, the case presents the intracranial infection caused by both Prevotella intermedia and Streptococcus constellatus which is of great clinical pressure due to its fatal consequences. Therefore, it is critical to receive early repeated imaging for patients with mixed infection meningitis accompanied with cerebral infarction; empiric antibiotic therapy should consider aerobic and anaerobic bacteria infection when brain diseases occur due to unknown reasons with turbid and stench CSF. In this sense, it is important to identify and diagnose mixed infectious meningitis at early stage using CSF culture when the patient is highly suspected with specific syndromes of this infection, especially acute exacerbating meningitis.

Acknowledgements we owe our special thanks to Yujie Huang for her assistance in revising this manuscript.

\section{Compliance with ethical standards}

Conflict of interest The authors declare that they have no conflict of interest.

Informed consent Informed consent of clinical studies was obtained from the patient and his family.

Animal studies and human participants All procedures performed in studies involving human participants were approved by the Institutional Ethical Review Board from the Fourth Affiliated Hospital of Guangxi Medical University (IRB No. LW2017003) in accordance with the ethical standards of the institute.

Open Access This article is distributed under the terms of the Creative Commons Attribution 4.0 International License (http:// creativecommons.org/licenses/by/4.0/), which permits unrestricted use, distribution, and reproduction in any medium, provided you give appropriate credit to the original author(s) and the source, provide a link to the Creative Commons license, and indicate if changes were made.

\section{References}

Aldridge KE (1995) The occurrence, virulence, and antimicrobial resistance of anaerobes in polymicrobial infections. Am J Surg 169:2s$7 \mathrm{~s}$

Bancescu G, Didilescu A, Bancescu A, Bari M (2015) Antibiotic susceptibility of 33 Prevotella strains isolated from Romanian patients with abscesses in head and neck spaces. Anaerobe 35:41-44

Baron EJ et al (2013) Executive summary: a guide to utilization of the microbiology laboratory for diagnosis of infectious diseases: 2013 recommendations by the Infectious Diseases Society of America (IDSA) and the American Society for Microbiology (ASM)(a). Clinical infectious diseases : an official publication of the Infectious Diseases Society of America 57:485-488

Brook I (2003) Prevotella intermedia meningitis associated with cerebrospinal fluid leakage in an adolescent. Pediatr Infect Dis J 22:751753

Brook I, Frazier EH (2003) Immune response to Fusobacterium nucleatum and Prevotella intermedia in the sputum of patients with acute exacerbation of chronic bronchitis. Chest 124:832-833

Chang WN, Lu CH, Huang CR, Chuang YC (2000) Mixed infection in adult bacterial meningitis. Infection 28:8-12

Chang WN, Wu JJ, Huang CR, Tsai YC, Chien CC, Lu CH (2002) Identification of viridans streptococcal species causing bacterial meningitis in adults in Taiwan. European journal of clinical microbiology \& infectious diseases : official publication of the European Society of Clinical Microbiology 21:393-396 
Chang WN, Lu CH, Huang CR, Tsai NW, Chuang YC, Chang CC, Chen SF, Chien CC (2008) Changing epidemiology of adult bacterial meningitis in southern taiwan: a hospital-based study. Infection 36: $15-22$

Contreras M, Costello EK, Hidalgo G, Magris M, Knight R, DominguezBello MG (2010) The bacterial microbiota in the oral mucosa of rural Amerindians. Microbiology (Reading, England) 156:32823287

Dubreuil L, Behra-Miellet J, Vouillot C, Bland S, Sedallian A, Mory F (2003) Beta-lactamase production in Prevotella and in vitro susceptibilities to selected beta-lactam antibiotics [corrected]. Int J Antimicrob Agents 21:267-273

Durand ML, Calderwood SB, Weber DJ, Miller SI, Southwick FS, Caviness VS Jr, Swartz MN (1993) Acute bacterial meningitis in adults. A review of 493 episodes. N Engl J Med 328:21-28

Frat JP, Godet C, Grollier G, Blanc JL, Robert R (2004) Cervical spinal epidural abscess and meningitis due to Prevotella oris and Peptostreptococcus micros after retropharyngeal surgery. Intensive Care Med 30:1695

Ganeshalingham A, Buckley D, Shaw I, Freeman JT, Wilson F, Best E (2014) Bacteroides fragilis concealed in an infant with Escherichia coli meningitis. J Paediatr Child Health 50:78-80

Jacinto RC, Gomes BP, Ferraz CC, Zaia AA, Filho FJ (2003) Microbiological analysis of infected root canals from symptomatic and asymptomatic teeth with periapical periodontitis and the antimicrobial susceptibility of some isolated anaerobic bacteria. Oral Microbiol Immunol 18:285-292

Ko JH et al (2015) Bacteremic meningitis caused by Parvimonas micra in an immunocompetent host. Anaerobe 34:161-163

Lai WA, Lu CH, Chang WN (2013) Mixed infection in adult postneurosurgical bacterial meningitis: a hospital-based study. Biom J 36:295-303

Leung KP, Fukushima H, Nesbitt WE, Clark WB (1996) Prevotella intermedia fimbriae mediate hemagglutination. Oral Microbiol Immunol 11:42-50

Llitjos JF, Mongardon N, Loubinoux J, Sonneville R, Charpentier J, Pene F, Mira JP (2016) Polymicrobial anaerobic meningitis. Medecine et maladies infectieuses 46:230-232

Moller K, Frederiksen EH, Wandall JH, Skinhoj P (1999) Meningitis caused by streptococci other than Streptococcus pneumoniae: a retrospective clinical study. Scand J Infect Dis 31:375-381
Mombelli A, Schmid B, Rutar A, Lang NP (2000) Persistence patterns of Porphyromonas gingivalis, Prevotella intermedia/nigrescens, and Actinobacillus actinomyetemcomitans after mechanical therapy of periodontal disease. J Periodontol 71:14-21

Okui H, Fukasawa C, Tokutake S, Takei H, Sato J, Hoshino T (2016) Pediatric Patient with anaerobic Bacterial Meningitis Who was Infected through a Spinal Congenital Dermal Sinus Route. Kansenshogaku zasshi The Journal of the Japanese Association for Infectious Diseases 90:321-324

Piscitelli SC, Shwed J, Schreckenberger P, Danziger LH (1992) Streptococcus milleri group: renewed interest in an elusive pathogen. European journal of clinical microbiology \& infectious diseases : official publication of the European Society of Clinical Microbiology 11:491-498

Roca B, Romero A, Simon E (1998) Acute meningitis caused by Streptococcus constellatus. The Journal of infection 37:88

Ruef C (2000) Bacterial meningitis-problems on many fronts. Infection $28: 1-2$

Sakamoto H, Karakida K, Otsuru M, Arai M, Shimoda M (2009) A case of brain abscess extended from deep fascial space infection. Oral Surg. Oral Med. Oral Pathol. Oral Radiol. Endod. 108:e21-e25

Shah HN, Collins DM (1990) Prevotella, a new genus to include Bacteroides melaninogenicus and related species formerly classified in the genus Bacteroides. Int J Syst Bacteriol 40:205-208

Sherrard LJ et al (2014) Mechanisms of reduced susceptibility and genotypic prediction of antibiotic resistance in Prevotella isolated from cystic fibrosis (CF) and non-CF patients. J Antimicrob Chemother 69:2690-2698

Tsai WC, Chen SF, Chang WN, Lu CH, Chuang YC, Tsai NW, Chang CC, Chien CC, Huang CR (2012) Clinical characteristics, pathogens implicated and therapeutic outcomes of mixed infection in adult bacterial meningitis. Kaohsiung J Med Sci 28:531-537

Weintraub A, Rashid MU, Nord CE (2016) In-vitro activity of solithromycin against anaerobic bacteria from the normal intestinal microbiota. Anaerobe 42:119-122

Wu PC, Tu MS, Lin PH, Chen YS, Tsai HC (2014) Prevotella brain abscesses and stroke following dental extraction in a young patient: a case report and review of the literature. Intern Med (Tokyo, Japan) 53:1881-1887 